\title{
CDF II Detector and Trigger for B Physics
}

\author{
S. Donati ${ }^{\mathrm{a}}$ \\ ${ }^{a}$ Istituto Nazionale di Fisica Nucleare, Sezione di Pisa, Edificio C - Polo Fibonacci, Largo B. \\ Pontecorvo, 3 - 56127 Pisa, Italy
}

In this paper we will review the elements of the CDF detector and trigger most relevant for B physics analyses.

\section{INTRODUCTION}

B hadrons are abundantly produced at the Tevatron Collider, the measured $\mathrm{B}^{+}$cross section is $3.6 \pm 0.6 \mu \mathrm{b}$ in the region of transverse momentum $p_{T}\left(B^{+}\right)>6.0 \mathrm{GeV} / \mathrm{c}$ and rapidity $\left|\eta\left(B^{+}\right)\right|<1$ [1]. This cross section is three orders of magnitude larger than at $e^{+} e^{-}$machines running at the $\Upsilon(4 S)$ and the available energy allows the production of the heavier $B_{s}^{0}, B_{c}$ and $\Lambda_{b}$ hadrons. The challenge is extracting the interesting B signals from a level of background which is three orders of magnitude higher at production. This is achieved at CDF II with dedicated detectors and triggers.

\section{THE TEVATRON COLLIDER}

The Tevatron Collider collides $36 p \bar{p}$ bunches at $\sqrt{s}=1.96 \mathrm{TeV}$. The design instantaneous luminosity was $10^{32} \mathrm{~cm}^{-2} \mathrm{~s}^{-1}$ but the Tevatron now exceeds it and set the peak luminosity record at $1.8 \times 10^{32} \mathrm{~cm}^{-2} \mathrm{~s}^{-1}$. With an already integrated luminosity of $1.3 \mathrm{fb}^{-1}$, the expectation is to have integrated $\sim 8 \mathrm{fb}^{-1}$ by the year 2008 .

\section{THE CDF II DETECTOR}

\subsection{The tracking system}

The CDF II tracker is located within a 14.1 $\mathrm{kG}$ solenoidal magnetic field and it is composed of silicon detectors and a drift chamber. There are three independent silicon detectors, SVXII, ISL and L00, for a total of eight silicon layers, 704 ladders and 722,432 channels [2]. SVXII is made of 360 double-sided ladders in a layout of six $15 \mathrm{~cm}$ axial sections $\times$ twelve $30^{\circ} \phi$ slices $\times$ five radial layers between 2.5 and $10.6 \mathrm{~cm}$ from the beamline. ISL covers the area between SVXII and the drift chamber, with 296 double-sided ladders at radii of 20 and $28 \mathrm{~cm}$. With a length of $1.9 \mathrm{~m}$, it provides silicon hits out to $|\eta|<2$. L00 is a single-sided layer of 48 ladders mounted directly on the beampipe, $1.5 \mathrm{~cm}$ from the beamline, which enhances the track impact parameter resolution. The three subdetectors share the same readout system, starting with the SVX3D chip, a custom designed ASIC with a 128 channel $\times 42$ capacitor analog storage ring, which makes it possible to acquire data in deadtimeless mode, integrating charge on one capacitor while reading out another one. The data acquisition system provides silicon data in time for Level 2 trigger processing. Over $90 \%$ of the silicon detector is powered and more than $80 \%$ is providing quality data. Charge collection efficiency is $99 \%$, with a single hit efficiency $>90 \%$. The hit resolution for a two-strip cluster is $9 \mu \mathrm{m}$. The signal-tonoise ratio is above 10:1 both for $r-\phi$ strips and for $r-z$ strips.

The Central Outer Chamber (COT, [3]) is located outside the silicon detectors and inside the time-of-flight detector scintillators. The active volume of the COT spans $310 \mathrm{~cm}$ in the beam direction, $43.4 \mathrm{~cm}$ and $132.3 \mathrm{~cm}$ in radius, and the entire azimuth. The COT contains 30,240 sense wires that run the length of the chamber between two end plates. Approximately half of the wires are axial (run along the $z$ direction) and half are small angle $\left(2^{\circ}\right)$ stereo. The $r-\phi$ view provides information for the $p_{T}$ measurement, the $r-z$ 
view for the $\eta$ measurement. The COT contains 96 sense wire layers in radius that are grouped into eight superlayers. Each superlayer is divided into supercells along the azimuthal angle, and each supercell has 12 sense wires and a maximum drift distance that is approximately the same for all superlayers. Therefore the number of supercells in a given superlayer scales approximately with the radius of the superlayer. The supercell layout consists of a wire plane containing sense and potential (or field shaping) wires and a field (or cathode) sheet on either side. Each field sheet is shared with the neighboring cell. The supercell is tilted by $35^{\circ}$ with respect to the radial direction to compensate for the Lorentz angle of the drifting electrons in the magnetic field. The gas is a mixture or Ar/Et (50:50) and Isopropyl which provides a maximum drift time of $177 \mathrm{~ns}$ on the maximum drift distance of $0.88 \mathrm{~cm}$. The measurement of the pulse widths provides a measurement of the $\mathrm{dE} / \mathrm{dx}$ in the chamber, used for particle identification. The achieved performance of the integrated CDF II tracker is a transverse momentum resolution $\sigma\left(p_{T}\right) / p_{T}^{2}=0.15 \%(\mathrm{GeV} / \mathrm{c})^{-1}$ and an impact parameter resolution $\sigma(d)=35$ $\mu \mathrm{m} @ 2 \mathrm{GeV} / \mathrm{c}$. This performance is sufficient for the $\mathrm{B}$ physics analyses.

\subsection{Particle identification detectors}

CDF II uses two detectors and two complementary techniques for particle identification, one is the $\mathrm{dE} / \mathrm{dx}$ measurement in the COT, the other one is the time-of-flight measurement in a dedicated detector. The COT readout electronics allows to measure the pulse width, which is related to the amount of charge collected by the wire. The truncated mean $(80 \%)$ computed on the hits associated to a track provides a measurement of the specific ionisation $(\mathrm{dE} / \mathrm{dx})$ in the chamber. A detailed calibration of the $\mathrm{dE} / \mathrm{dx}$ measurement has been performed using samples of kaons and pions from $D^{*+} \rightarrow D^{0} \pi^{+} \rightarrow\left[K^{-} \pi^{+}\right] \pi^{+}$, protons from $\Lambda^{0} \rightarrow p \pi^{-}$, and muons and electrons from $J / \psi \rightarrow \mu^{+} \mu^{-}$and $J / \psi \rightarrow e^{+} e^{-}$. The achieved $K / \pi$ separation for $p_{T}>2 \mathrm{GeV} / \mathrm{c}$ is $1.4 \sigma$.

The Time-of-Flight detector (TOF, [4]) is istalled between the drift chamber and the solenoid magnet and extends $4.7 \mathrm{~cm}$ radially at a radius of roughly $138 \mathrm{~cm}$. The detector is composed by Bicron scintillator bars BC-408, selected for the long $(2.5 \mathrm{~m})$ attenuation length. The bars have a dimension of $4 \times 4 \mathrm{~cm}^{2}$ in cross-section and $279 \mathrm{~cm}$ in length. There are a total of 216 bars, each covering $1.7^{\circ}$ in $\phi$ and $|\eta|<1$. The photomultipliers are attached to each end of every bar. The time resolution on the single hit is $110 \mathrm{ps}$ and the $K / \pi$ separation is better than $2 \sigma$ for $p_{T}<1.5 \mathrm{GeV} /$ c. By combining the $\mathrm{dE} / \mathrm{dx}$ and the time-of-flight measurements, the achieved $K / \pi$ separation is better tha $1.4 \sigma$ in the entire momentum range.

\subsection{Lepton detectors}

Segmented electromagnetic and hadronic calorimeters surround the tracking system [6]. The electron energy is measured by leadscintillator sampling calorimeters. In the central region $(|\eta|<1.1)$ the calorimeters are arranged in a projective barrel geometry and measure electromagnetic energy with a resolution of $\left|\sigma\left(E_{T}\right) / E_{T}\right|^{2}=(13.5 \%)^{2} / E_{T}(\mathrm{GeV})+(2 \%)^{2}$. In the forward region $(1.2<|\quad \eta \quad|<3.5)$ the calorimeters are arranged in a projective endplug geometry and measure the electromagnetic energy with a resolution of $\left|\sigma\left(E_{T}\right) / E_{T}\right|^{2}=$ $(14.4 \%)^{2} / E_{T}(\mathrm{GeV})+(0.7 \%)^{2}$. Both central and forward electromagnetic calorimeters are instrumented with finely segmented detectors which measure the shower position at a depth where the energy deposition by a typical shower reaches its maximum. The central muon detector (CMU [5]) is located around the outside of the central hadron calorimeter at a radius of $347 \mathrm{~cm}$ from the beam axis. The calorimeter has a thickness of 5.5 interaction lengths and a $\phi$ segmentation of $15^{\circ}$. The muon drift cells are $226 \mathrm{~cm}$ long and cover $12.6^{\circ}$ in $\phi$, giving a $\phi$ coverage of $84 \%$. The pseudorapidity coverage is $|\eta|<1$. Each module consists of four layers of four rectangular drift cells. The sense wires in alternating layers are offset by $2 \mathrm{~mm}$ for ambiguity resolution. The smallest unit in the CMU, called a stack, covers about $1.2^{\circ}$ and includes four drift cells, one from each layer. Adjacent pairs of stacks are combined together to form a two-stack unit called a tower. A track segment detected in these chambers is 
called a CMU stub. A second set of muon chambers is located behind an additional $60 \mathrm{~cm}$ of steel. The chambers are arranged axially to form a box around the central detector. The coverage of the central muon system is extended to the region $0.6<|\eta|<1.0$ by four free-standing conical arches which hold drift chambers which cover $71 \%$ of the solid angle.

\section{THE CDF II TRIGGER}

CDF II uses a three-level system to reduce the 1.7 $\mathrm{MHz}$ bunch crossing rate to $100 \mathrm{~Hz}$ written on tape. The Level 1 is a deadtimeless $7.6 \mathrm{MHz}$ synchronous pipeline with 42 cells, which allows $5.5 \mu \mathrm{s}$ to form a trigger decision. The maximum sustainable Level 1 output rate is $30 \mathrm{kHz}$. The Level 2 is an asynchronous pipeline with an average latency of $20 \mu \mathrm{s}$. While the events accepted by Level 1 are being processed by Level 2 processors, they are also stored on one of the four Level 2 buffers, waiting for Level 2 trigger decision. Each buffer is emptied when the Level 2 decision for the corresponding event has been asserted: if the event has been accepted, the buffer is read out, else it is simply cleared. If the Level 2 trigger decision takes too much time and the four buffers are all filled, the Level 1 accept is inhibited. This is a source of deadtime for the CDF II trigger. The maximum Level 2 output rate is $300 \mathrm{~Hz}$. The Level 3 trigger is made of a CPU farm and has a maximum output rate of $100 \mathrm{~Hz}$.

The heart of the Level 1 trigger is the eXtremely Fast Tracker (XFT, [7]), the trigger track processor that identifies high transverse momentum $\left(p_{T}>1.5 \mathrm{GeV} / \mathrm{c}\right)$ charged tracks in the transverse plane of the COT. The XFT tracks are also extrapolated to the calorimeter and to the muon chambers to generate electron and muon trigger candidates. Track identification in the XFT is accomplished in two processes by the Finder and by the Linker. The Finder searches for track segments in the four axial superlayers of the chamber. The Linker searches for a four-out-offour or a three-out-of-four match among segments in the four layers, consistent with a prompt high$p_{T}$ track. The efficiency for finding XFT tracks is $96 \%$, with a transverse momentum resolution better than $2 \%$ per $\mathrm{GeV} / \mathrm{c}$ and azimuthal angular resolution of $5.5 \mathrm{mr}$. The level of fake tracks is $3 \%$ at luminosity below $5 \times 10^{31} \mathrm{~cm}^{-2} \mathrm{~s}^{-1}$ and grows with instantaneous luminosity.

The Online Silicon Vertex Tracker (SVT, [8]) is part of the Level 2 trigger. It receives the list of XFT tracks and the digitised pulse heights on the axial layers of the silicon vertex detector. The SVT links the XFT tracks to the silicon hits and reconstructs tracks with offline-like quality. In particular the resolution on the impact parameter, which is a crucial parameter to select B events since they tipically show secondary vertices, is $35 \mu \mathrm{m}$ for $2 \mathrm{GeV} / \mathrm{c}$ tracks. The SVT efficiency is $85 \%$ per track. Since a long Level 2 processing time can introduce dead time, to speed up operations the SVT has a widely parallelized design: it is made of 12 identical azimuthal slices working in parallel. Each slice receives and processes data only from one silicon vertex detector $30^{\circ}$ sector. In addition SVT recontructs only tracks in the transverse plane to the beamline and only with $p_{T}>2.0 \mathrm{GeV} / \mathrm{c}$. The tracking process is performed in two steps. The first step is the pattern recognition: candidate tracks are searched among a list of precalculated low resolution patterns. This is done in order to reduce the huge amount of silicon hits only to those potentially interesting. The second step is track fitting: a full resolution fit of the hit coordinates found within each pattern is performed using a linearized algorithm. By providing a precision measurement of the impact parameter of the charged particle tracks, SVT allows triggering on events cointaining long lived particles, like the B events, which at the Tevatron have decay lengths of the order of $500 \mu \mathrm{m}$ and produce tracks in the decay with impact parameters on average larger than $100 \mu \mathrm{m}$.

Level 3 trigger is implemented on a CPU farm which allows to perform an almost offline-quality event reconstruction.

\subsection{Triggers for $B$ physics}

CDF II has basically three families of triggers for B physics: the dimuon trigger, the semileptonic trigger and the hadronic trigger.

The dimuon trigger selects muon pairs with transverse momenum as low as $1.5 \mathrm{GeV} / \mathrm{c}$. It is 
mostly used to select $J / \psi \mathrm{s}$ and $\psi(2 S)$, to reconstruct the many decay modes of the B hadrons $\left(B^{0}, B^{+}, B_{s}^{0}, B_{c}\right.$, and $\left.\Lambda_{b}\right)$ containing a $J / \psi$ decaying to muon pairs, and to select $\Upsilon \rightarrow \mu^{+} \mu^{-}$ decays, or muon pairs for the search of the rare $B \rightarrow \mu^{+} \mu^{-} X$ decays, or for $b \bar{b}$ correlation studies.

The semileptonic trigger selects events with a lepton $\left(\mu\right.$ or $e$ ) with $p_{T}>4 \mathrm{GeV} / \mathrm{c}$ and an SVT track with $p_{T}>2 \mathrm{GeV} / \mathrm{c}$ and impact parameter above $120 \mu \mathrm{m}$.

The hadronic trigger selects track pairs with $p_{T}>2 \mathrm{GeV} / \mathrm{c}$ and $p_{T 1}+p_{T 2}>5.5 \mathrm{GeV} / \mathrm{c}$, with an opening angle in the transverse plane below $135^{\circ}$, impact parameter above $100 \mu \mathrm{m}$, a decay length above $200 \mu \mathrm{m}$. For the two-body decay trigger path, optimised to collect $B \rightarrow h^{+} h^{\prime}-$ decays, the track pair is requested to point back to the primary vertex, by requiring that the impact parameter of the reconstructed $\mathrm{B}$ is below $140 \mu \mathrm{m}$. To select hadronic multibody decays, like $B_{s}^{0} \rightarrow D_{s}^{-} \pi^{+}$, the request on the pointing back to the primary vertex has low efficiency, since the track pair provides only a partial reconstruction of the multibody decay, and it is not applied.

The Tevatron performance has been continuosly improving since the beginning of Run II. At the time of writing these proceedings, the initial instantaneous luminosity of the stores may reach $1.8 \times 10^{32} \mathrm{~cm}^{-2} \mathrm{~s}^{-1}$, which is almost a factor of of two above design luminosity $\left(10^{32} \mathrm{~cm}^{-2} \mathrm{~s}^{-1}\right)$, and the stores can last up to 30 hours, when they are dropped at an instantaneous luminosity around $2 \times 10^{31} \mathrm{~cm}^{-2} \mathrm{~s}^{-1}$. This is a factor of 9 decrease with respect to the initial store luminosity. If the trigger bandwidth is filled at the initial luminosity, it will get less and less occupied as the store luminosity decreases. So the problem is to adjust the trigger selections in order to have all the available bandwidth completely used at all instantaneous luminosities. This is achieved by implementing more versions of the same trigger with increasingly tighter cuts, and consequently increasing purity, and running the versions with tighter cuts with no prescale and the versions with looser cuts with a prescale. For the hadronic trigger the tighter version requires track pairs with $p_{T}>2.5 \mathrm{GeV} / \mathrm{c}$ and $p_{T 1}+p_{T 2}>6.5 \mathrm{GeV} / \mathrm{c}$.
The prescales can be made dynamical, and they adjust themselves as the luminosity decreases and more bandwidth becomes available. In addition to this, triggers are luminosity enabled: if the rate of a trigger that is important to keep unprescaled is too large at the initial luminosity, it is enabled only when the luminosity has decreased below a safe threshold.

\subsection{Trigger upgrades}

The CDF II trigger was designed for an instantaneous luminosity of $10^{32} \mathrm{~cm}^{-2} \mathrm{~s}^{-1}$. The increase above this limit already achieved and the expectation to reach $3 \times 10^{32} \mathrm{~cm}^{-2} \mathrm{~s}^{-1}$ demanded an upgrade of the system. The increased occupancy of the detectors, in particular of the COT, results in the increase of the rate of fake tracks in XFT, and consequently in the increase of the Level 1 triggers which use XFT tracks. The increased complexity of the events deriving from the pile up of more interactions in the same bunch crossing, results in the increase of the SVT processing time and consequently in the increase of the trigger deadtime. The XFT is being upgraded to perform a three dimensional track recontruction and the SVT has been upgraded to use faster and more performant components.

\section{REFERENCES}

1. D. Acosta et al., the CDF Collaboration, Phys. Rev. D 65, 052005 (2002).

2. A. Sill et al., Nucl. Instrum. Meth. A530:1 (2004).

3. T. Affolder et al., Nucl. Instrum. Meth. A526:249 (2004).

4. D. Acosta et al., Nucl. Instrum. Meth. A518:605 (2004).

5. G. Ascoli et al., Nucl. Instrum. Meth. A268:33 (1988).

6. L. Balka et al., Nucl. Instrum. Meth. A267:272 (1988). M. Alborw. et al., Nucl. Instrum. Meth. A453:84 (2000).

7. E. J. Thomson et al., IEEE Trans. on Nucl. Sc. vol. 49, n. 3 (2002).

8. A. Bardi et al., Nucl. Instrum. Meth. A485:178 (2002). 